\title{
Precision Control of Eluted Activity from a Sr/Rb Generator for Cardiac Positron Emission Tomography
}

\author{
R. Klein ${ }^{1}$, A. Adler ${ }^{2}$, R. S. Beanlands ${ }^{1}$, R. A. deKemp ${ }^{1}$ \\ ${ }^{1}$ National Cardiac PET Centre, University of Ottawa Heart Institute, Ottawa, Ontario, Canada \\ ${ }^{2}$ School of Information Technology and Engineering, University of Ottawa, Ontario, Canada
}

\begin{abstract}
A rubidium-82 $\left({ }^{82} \mathbf{R b}\right)$ elution system is described for use with clinical positron emission tomography. The system is self-calibrating with $1.4 \%$ repeatability, independent of generator activity and elution flow rate. Saline flow is switched between a ${ }^{82} \mathrm{Sr} /{ }^{82} \mathrm{Rb}$ generator and a bypass line to achieve a constant activity elution of ${ }^{82} \mathrm{Rb}$. In the present study, pulse width modulation (PWM) of a solenoid valve is compared to simple threshold control as a means to simulate a proportional valve. A predictive-corrective control algorithm is developed which produces a constant activity elution within the constraints of long feedback delay and short elution time. Accurate constant-activity elutions of $10-70 \%$ of the total generator activity were demonstrated using the threshold comparison control. The adaptive-corrective control of the PWM valve provided a substantial improvement in precision of the steady-state output.
\end{abstract}

Keywords - Strontium-82 Rubidium-82 generator, elution system control, cardiac PET imaging

\section{INTRODUCTION}

Myocardial perfusion imaging with the radiotracer rubidium-82 $\left({ }^{82} \mathrm{Rb}\right)$ and positron emission tomography (PET) is used commonly to diagnose coronary artery disease. Absolute measurements of perfusion can also be made from the rate of tracer uptake using dynamic PET imaging [1]. To facilitate reproducible measurements, the tracer should be introduced at a constant rate over a short period of time $(30 \mathrm{~s}) .{ }^{82} \mathrm{Rb}$ is produced from a ${ }^{82} \mathrm{Sr} /{ }^{82} \mathrm{Rb}$ generator and has a very short half-life $(76 \mathrm{~s})$. Therefore it requires direct intravenous infusion to the patient with an automated generator elution system [2].

${ }^{82} \mathrm{Sr} /{ }^{82} \mathrm{Rb}$ generators have been described by our group and others for the production of ${ }^{82} \mathrm{Rb}[3,4]$. The generator is an ion-exchange column of tin-oxide that binds the parent isotope ${ }^{82} \mathrm{Sr}$ (25 day half-life) but not the daughter ${ }^{82} \mathrm{Rb}$ resulting from ${ }^{82} \mathrm{Sr}$ decay. Therefore ${ }^{82} \mathrm{Rb}$ is eluted from the generator simply by flushing with $0.9 \%$ saline. In the generator column, ${ }^{82} \mathrm{Rb}$ exists at equilibrium levels with ${ }^{82} \mathrm{Sr}$ and regenerates within 10 minutes after elution, making this tracer convenient for rapid serial perfusion studies.

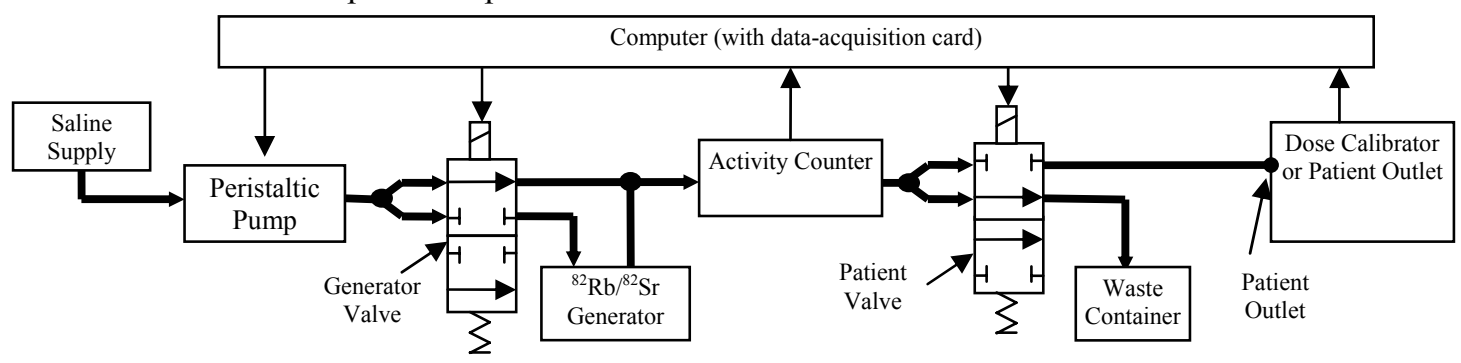
activity elutions from the ${ }^{82} \mathrm{Rb}$ generator.

\section{DESIGN}

\section{A. System Description} waste container. readings to ${ }^{82} \mathrm{Rb}$ activity in $\mathrm{Bq} / \mathrm{ml}$.
Fig. $1-{ }^{82} \mathrm{Rb}$ elution system diagram showing saline line (thick lines), control signals, sensor signals, and major components. repeatable and characteristic curve of activity vs. volume over a wide range of flows [2], whereas the eluted activity vs. time is dependent on the saline flow rate [5]. The scale of the characteristic curve depends on the amount of ${ }^{82} \mathrm{Sr}$ in the column, and the shape is characterized by an initial peak of high activity as the volume of the generator column is flushed (bolus stage) followed by an asymptotic decrease to an equilibrium of ${ }^{82} \mathrm{Rb}$ production, flushing, and decay (continuous stage). The purpose of this study was to investigate the use of feedback control to obtain constant-

A bypass line of the generator is used with a single twoway solenoid pinch valve to direct flow through the generator or to bypass it (Fig.1). The lines are merged immediately upstream from an activity (positron) counter. The line volume between the generator and the activity counter is kept to a minimum, while ensuring significant shielding to reduce background counts from the generator. Downstream from the counter an additional two way pinch valve routes the elution to the patient outlet or to a shielded

A constant flow rate $\mathrm{F}$ is set with a peristaltic pump. This allows for prediction of the activity at the patient outlet, $\mathrm{A}_{\text {pat }}$, after a transmission delay of $\mathrm{D}$ seconds, based on counts measured at the activity counter, $\mathrm{C}_{\mathrm{det}}$ using

$$
A_{p a t}(t+D)=C_{\mathrm{det}}(t) \cdot e^{-\lambda D} \cdot K
$$

where $\lambda=\ln (2) / 76$ is the ${ }^{82} \mathrm{Rb}$ decay constant. The calibration constant $\mathrm{K}$, accounting for the intrinsic and geometric efficiency of the activity counter is used to convert counter 


\section{B. Calibration}

An external dose calibrator (Capintec CRC-15R) is used as the gold standard for comparison of the accumulated counter readings both for calibration and testing. Calculation of the calibration constant was modified from that described in [2] using

$$
K=\frac{\sum \hat{A}_{c a l}(t)}{\sum A_{p a t}(t)}=\frac{\sum A_{c a l}(t-\tau) \cdot e^{-\lambda \tau}}{\sum\left(C_{\mathrm{det}}(t) \otimes e^{-\lambda t}\right) F \cdot e^{-\lambda D}}
$$

The effective delay from the activity counter to the dose calibrator, $\tau$, was determined through minimization of the mean square error between the calibrator readings, $\hat{\mathrm{A}}_{\text {cal }}$, and the predicted accumulated activity in the vial, $A_{\text {pat }}$.

Calibration runs were carried out at a flow rate of $15 \mathrm{ml} / \mathrm{min}$ over 60 seconds. Over the entire elution and the following 120 seconds, readings from the activity counter and the dose calibrator were recorded simultaneously at 1 second intervals. Plotting of $\hat{\mathrm{A}}_{\text {cal }}$ and $\mathrm{A}_{\mathrm{pat}}$ reveals slightly different shapes of the calibrator vs. detector curves (Fig.2). This was determined to be due to detection of activity in the patient line upstream from the patient outlet. An additional convolution kernel was created to describe this geometric aperture. The kernel was estimated to have the shape of a normalized Gaussian over the interval $\mid-\infty, 0]$ with the width adjusted for the geometry of the dose calibrator.

$$
G(t)=c e^{-\gamma(F t)^{2}}
$$

The parameter $\gamma$ was adjusted emperically based on a series of calibration runs at $5-25 \mathrm{ml} / \mathrm{min}$. The resulting plots corresponded more closely in shape, especially at low flow rates of $5-10 \mathrm{ml} / \mathrm{min}$.

\section{Constant Activity Elution and Control Algorithms}

The readings from the activity counter are used as feedback to a real-time control system, which controls the pinch valve to achieve a constant activity rate. Initially, the patient valve routes the elution to the waste until the threshold activity is reached (Fig. 3). It is switched by the control system to the patient outlet after the flow delay from the activity counter to the patient valve. This removes the initial transient rise of activity to produce a step response at the patient outlet. The elution continues until the desired total activity has been produced. At the end of the elution the generator valve is closed creating a step drop in output. The elution of saline is continued through the bypass line to flush the activity from the counter to the patient outlet.

Two approaches to control the generator/bypass valve were explored. The threshold comparison approach controls the valve through comparison of the instantaneous activity rate to the set point. This results in activity fluctuations around the desired set point. The set point was compensated for hysteresis using a single factor that was empirically determined and verified to be 1.2 and is independent of flow rate.
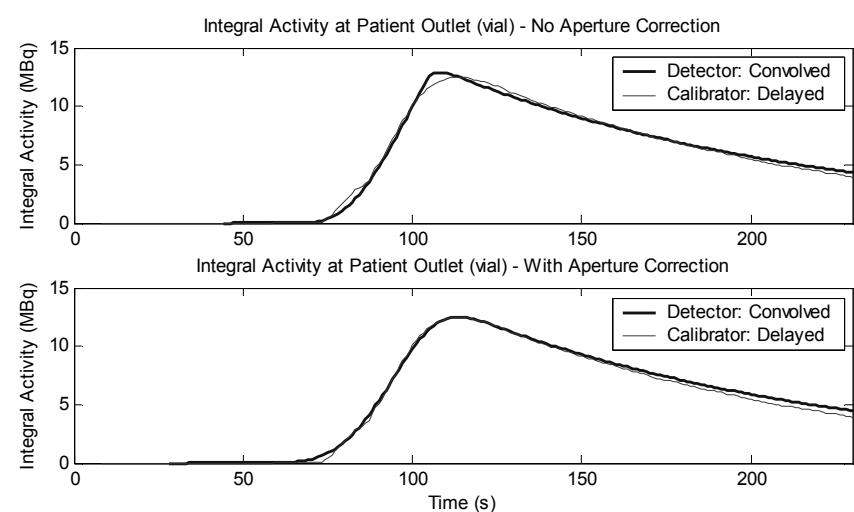

Fig. 2 - Integral activity at the vial as measured by the dose calibrator (thin line) and estimated from the activity counter (thick line). The lower graph demonstrates the improvement in the estimate due to compensation for the aperture of the dose calibrator $(\gamma=0.01)$. Flow rate is $5 \mathrm{ml} / \mathrm{min}$.

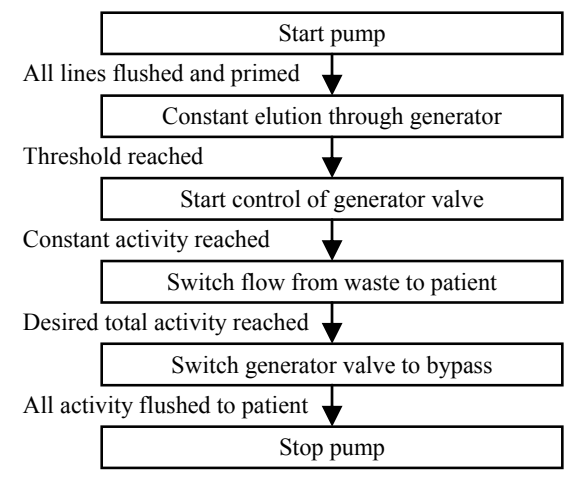

Fig. 3 - Constant activity elution sequence diagram.

A second, approach involved the simulation of a variable pinch valve by pulse-width-modulation (PWM) of the saline flow [6]. The valve was vibrated at $5 \mathrm{~Hz}$ and the duty cycle was controlled. A minimum 'on' time, $\mathrm{T}_{\min }$, is required to overcome the valve spring and cause it to move which limits the vibration frequency. The PWM was implemented in a single software block, and allowed us to develop the remaining control algorithms as if controlling a variable pinch valve (Fig. 4).

The traditional Proportional-Integral-Differential (PID) controller has some inherent shortcomings which make it inadequate for this application. These include a varying feedback delay due to different saline flow rates. In addition, a PID loop will always lag in response to the error as a result of feedback delay. Finally, the short elution time and low sampling frequency do not allow for long stabilization times.

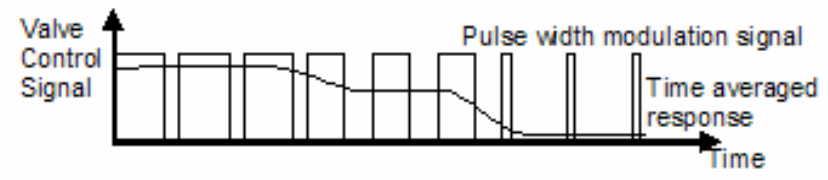

Fig. 4 - Pulse-width-modulation control of a solenoid valve to simulate a variable pinch valve. Flow through the generator increases as the duty cycle is increased. 
To overcome the shortcoming of the PID controller, a predictive-corrective controller was developed. The activity from the generator is predicted based on the activity-volume curves measured during the same day's calibration run. A ratio of the desired activity and the predicted instantaneous activity is used as an estimate of the position of the variable valve. A PID feedback loop uses the measured count errors to correct the valve position.

\section{PWM Controlled Valve Calibration}

At the end of an elution the discrepancy in elution time is used to adjust $T_{\min }$. This iterative process adapts the estimation of $T_{\min }$ over time. $T_{\min }$ is expected to change due to material fatigue, change of tubing and other system variations. In our system $T_{\min }$ converged to $162 \mathrm{~ms}$ within less than 20 elutions and has not changed significantly since.

Since the long $\mathrm{T}_{\min }$ corresponds to a minimum duty cycle of $80.1 \%$ a relatively small active range of the PWM is left for control. Since the implementation is in discrete time, this results in a reduced resolution of the control signal. The number of quantized steps, $\mathrm{Q}$, is given by (4) where $\mathrm{M}(\mathrm{Hz})$ is the software model refresh rate and $\mathrm{V}(\mathrm{Hz})$ is the vibration frequency. In our case the model is run at $2000 \mathrm{~Hz}$ resulting in 78 quantized steps.

$$
Q=\left\lfloor\frac{M}{V}\left(1-T_{\min } V\right)\right\rfloor
$$

\section{EXPERIMENTAL METHODS}

\section{A. Calibration}

Daily calibration runs were conducted as described above to test for repeatability of the calibration and test for variation over time. Additional calibration runs were carried out at flow rates ranging from 5 to $25 \mathrm{ml} / \mathrm{min}$ to test the accuracy of the physical model with changing flows.
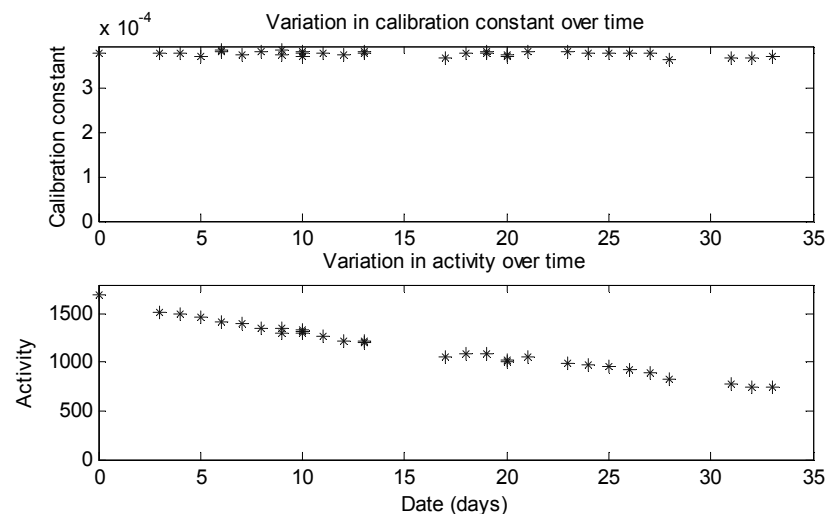

Fig. 5 - Calibration constant over the course of a generator life (top) and the dose from the generator during calibration over the same time period (bottom)

\section{B. Constant Activity Elutions}

A series of $30 \mathrm{~s}$ constant-activity elutions was carried out for desired output activity in the range of $10-70 \%$ of the total calibration activity. Output activity was measured relative to the calibration activity to accommodate for the $\mathrm{Sr}$ decay over the course of the study. Both threshold comparison control and adaptive-corrective controls were assessed for comparison.

\section{RESUlts}

\section{A. Calibration}

Over the life-span of one generator, 35 calibration runs were conducted at $15 \mathrm{ml} / \mathrm{min}$ flow rate (Fig. 5). The calibration constant appears unchanged with a standard deviation of $1.4 \%$ over time.

Calibration values were analyzed at flow rates ranging from $5-25 \mathrm{ml} / \mathrm{min}$ (Fig. 6). The value of the calibration constant did not vary over the range $10-25 \mathrm{ml} / \mathrm{min}$, but at 5 $\mathrm{ml} / \mathrm{min}$ decreased slightly. The reason for this variation has not been determined but is not of much concern as this low flow rate is an extreme case and is not typically used in clinical application.

\section{B. Constant Activity Elutions}

Table 1 shows the mean square error (MSE), elution time error, and dose error as a function of the desired relative dose. These data show the desired elution time is achieved using the threshold comparison control but fluctuations cause a $>30 \%$ mean squared error. The proposed control method improves the MSE at intermediate activities but performs less well at the extremes. These results are partly artifacts of the initial activity peak after the threshold activity is detected (Fig. 7). The source of this peak has not yet been confirmed, but is expected to be a sudden drop in back pressure as the patient valve is switched

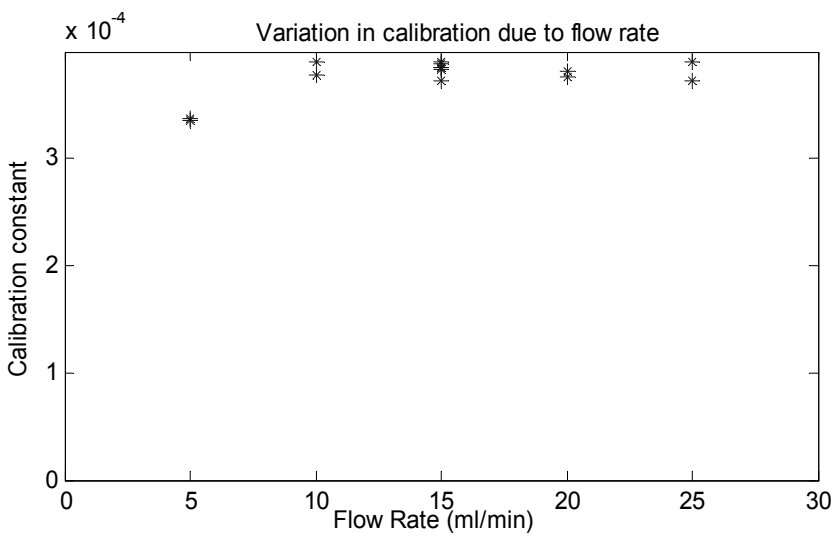

Fig. 6 - Calculated calibration constant over the range of flow rates. 
TABLE I

CONSTANT FLOW RATE RESULTS

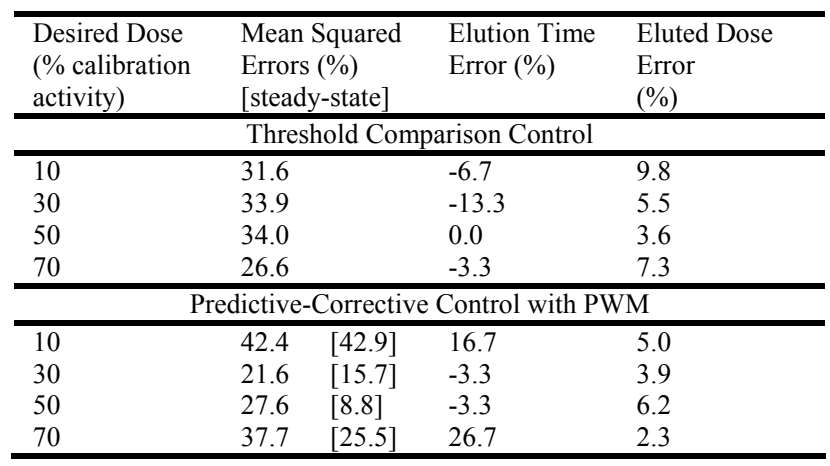
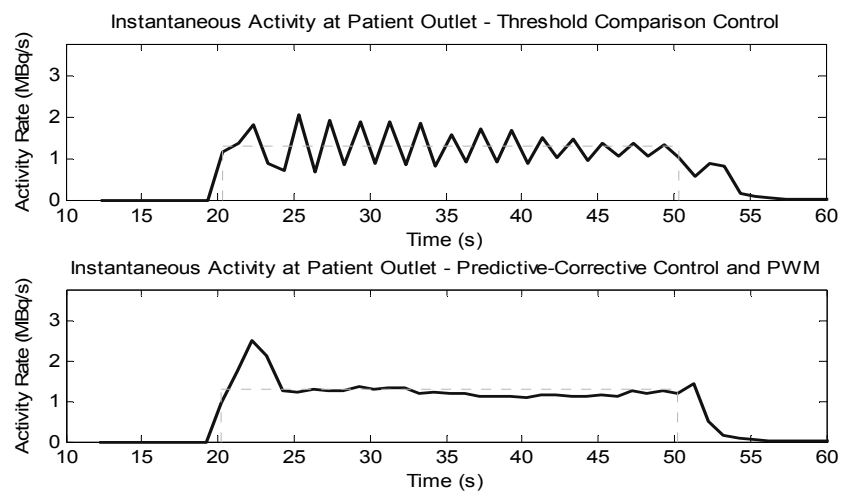

Fig. 7 - Similar elutions at 50\% generator capacity using the threshold comparison algorithm (top) and the adaptive-corrective control with PWM valve control (bottom). The dotted lines show the desired profile.

from waste to patient outlet, resulting in a surge of active saline from the generator. Fig. 7 demonstrates sample elutions of $50 \%$ generator activity. The threshold control results in a MSE of $34.0 \%$. With the exception of the initial peak during the first $4 \mathrm{~s}$, the steady state MSE is only $8.8 \%$ and results partly from correction of the initial peak.

As mentioned above, the high duty-cycle threshold, $\mathrm{T}_{\min }$, required to vibrate the valve significantly limits the resolution of variable valve implementation. To date we have implemented the vibration control in software. Future improvements would implement the PWM in hardware using a timer. With counters running in the $\mathrm{MHz}$ range, the control signal resolution would be 3 orders of magnitude higher than our current implementation, and may help to further reduce the steady state MSE.

\section{Breakthrough Sr Activity}

Elution of ${ }^{82} \mathrm{Sr}$ and ${ }^{85} \mathrm{Sr}$ isotopes (half-life 25 and 65 days respectively) to the patient is undesired, as $\mathrm{Sr}$ tends to accumulate in the bone marrow, which is particularly radiation sensitive.

Daily calibration samples are used to test for the breakthrough of Sr activity. Our generator is limited to $20 \mathrm{~L}$ of elution for clinical use. After expiry we continued testing and did not detect significant breakthrough activity. Even after exceeding this specification by approximately $50 \%$, breakthrough activity remained 15 and 13 times below the established breakthrough limits for ${ }^{82} \mathrm{Sr}$ and ${ }^{85} \mathrm{Sr}$ respectively. This leads us to believe that breakthrough is not significantly accelerated by using the feedback controlled elutions, but more experience is required for confirmation.

\section{CONCLUSION}

We have demonstrated a feedback control system for ${ }^{82} \mathrm{Rb} /{ }^{82} \mathrm{Sr}$ generators to achieve constant-activity elutions. In addition we have confirmed the understanding of the physical model through successful calibration at various flow rates and generator activity. Work to date indicates that using pulse-width-modulation to simulate a variable position valve will enable constant-activity elutions with improved precision. In addition a predictive-corrective algorithm is able to control the modulation, however, more work is still required to reduce the initial transient overshoot.

\section{ACKNOWLEDGMENT}

We would like to thank May Aung and Kimberly Gardner for their collaboration and feedback during the initial clinical trials of the system.

\section{REFERENCES}

[1] J. W. Lin, R. R. Sciacca, R. L. Chou, A. F. Laine, S. R. Bergmann, "Quantification of myocardial perfusion in human subjects using ${ }^{82} \mathrm{Rb}$ and wavelet-based noise reduction", J. Nucl. Med., Vol. 42, No. 2, February 2001.

[2] N. J. Epstein, A. Benelfassi, R. S. Beanlands, R. A. deKemp, “A ${ }^{82} \mathrm{Rb}$ infusion system for quantitative perfusion imaging in $3 \mathrm{D}$ PET", App. Radiat. Isot, In Press, 2004.

[3] T. M. Alvarez-Diez, R. A. deKemp, R. S. Beanlands, J. Vincent, "Manufacture of strontium- 82 /rubidium- 82 generators and quality control of rubidium- 82 chloride for myocardial perfusion imaging in patients using positron emission tomography", Appl. Radiat. Isot., Vol. 50, pp 1015-1023, 1999.

[4] Y. Yano, "Essentials of a Rubidium-82 generator for nuclear medicine", Appl. Radiat. Isot., Vol 38, No 3, pp. 205-211, 1987.

[5] V. Dhawan, "Model for ${ }^{82} \mathrm{Sr} /{ }^{82} \mathrm{Rb}$ generator elution profiles: A second approach to radioawway/dosimetry", Appl. Radiat. Isot., Vol. 38, No. 3, pp. 233-239, 1987.

[6] T. Imaizumi, O. Oyama, Yoshimitsu T., "Study of pneumatic servo system employing solenoid valve instead of proportional valve by keeping the solenoid valve plunger to be floating", Flucom Symposium Proceedings, article 076, 2000. 